\title{
Alimentação saudável e sustentável: uma revisão narrativa sobre desafios e perspectivas
}

\author{
Healthy and sustainable diet: a narrative review of the challenges \\ and perspectives
}

Suellen Secchi Martinelli (https://orcid.org/0000-0001-9263-0867) ${ }^{1}$

Suzi Barletto Cavalli (https://orcid.org/0000-0002-2835-9424) ${ }^{1}$

${ }^{1}$ Departamento de

Nutrição, Universidade Federal de Santa Catarina. R. Eng. Agronômico Andrei Cristian Ferreira s/n, Trindade. 88040-900 Florianópolis SC Brasil. suellen.martinelli@ufsc.br

\begin{abstract}
Based on the principle that a non-sustainable food system is not capable of producing healthy food for consumption, food can only be considered healthy if it is also sustainable, going beyond the nutritional perspective. Therefore, a narrative review of the scientific literature on the sustainable and health food system was conducted, regarding aspects of production, processing, marketing and consumption, seeking to pinpoint the challenges and perspectives for its consolidation. Food systems needs to be related to a food production and consumption system which protects biodiversity and promote a diverse consumption, bringing back traditional dishes and preparation techniques. It should also be accessible and available for everyone, both in quantity and in quality, based on food that is locally produced by family farmers through agroecology and founded on fair trade, bringing production and consumption closer together. In addition, it must be free from physical, biological or chemical contaminants that cause damage to everyone involved, whether it be an isolated incident or chronically. Key words Food production, Products commerce, Food-processing industry, Health behavior
\end{abstract}

Resumo Parte-se do princípio que um sistema alimentar insustentável não é capaz de produzir alimentos saudáveis para o consumo. A alimentação só pode ser considerada saudável se for também sustentável, devendo ultrapassar a perspectiva nutricional. Assim, realizou-se uma revisão narrativa de literatura acerca de sistemas alimentares saudáveis e sustentáveis, englobando aspectos de produção, processamento, comercialização e consumo, visando levantar seus desafios e perspectivas de consolidação. A alimentação saudável e sustentável deve estar relacionada à produção de alimentos que protejam a biodiversidade e promovam o consumo variado, resgatando alimentos, preparações e hábitos culturais tradicionais. Deve ser acessivel e disponivel a todos, em quantidade e qualidade, baseada em alimentos produzidos e processados na região, por agricultores familiares, de maneira agroecológica, fundamentada na comercialização justa, aproximando a produção do consumo. Além disso, deve ser isento de contaminantes físicos, biológicos ou químicos que causem malefícios a todos os envolvidos, de maneira aguda ou crônica.

Palavras-chave Produção de alimentos, Comercialização de produtos, Indústria de processamento de alimentos, Comportamentos saudáveis 


\section{Introdução}

As preocupações em relação à alimentação vêm se modificando com o passar do tempo. O médico e nutrólogo argentino Pedro Escudero recomendou, em 1934, que uma alimentação saudável fosse aquela qualitativamente completa, quantitativamente suficiente, harmoniosa em sua composição e apropriada à sua finalidade e a quem se destina ${ }^{1}$. Durante muito tempo, as preocupações em relação à alimentação estiveram centradas no elevado consumo de alimentos com alto teor de açúcar, sódio e gordura. As preocupações são pertinentes, já que o elevado consumo desses alimentos, aliado a fatores como sedentarismo e estresse, está relacionado à incidência de Doenças Crônicas Não Transmissíveis (DCNT), responsáveis por elevadas taxas de mortalidade da população nos últimos anos ${ }^{2}$. Considerando essas tendências, a Organização Mundial da Saúde (OMS) fez algumas recomendações por meio da Estratégia Global para Alimentação Saudável, Atividade Física e Saúde 3 . Vale ressaltar que as recomendações de Escudero, que datam de 1934 e que foram ratificadas pela Estratégia Global, continuam sendo importantes para a qualidade nutricional dos alimentos consumidos, mas é inegável a necessidade de incorporar demandas relacionadas à produção e processamento de alimentos, que na época não se faziam necessárias.

As alterações no sistema alimentar são recentes, mas, apesar disso, vêm causando danos sociais, econômicos e ambientais de forma crescente. Sistema alimentar refere-se ao conjunto de processos que incluem agricultura, pecuária, produção, processamento, distribuição, abastecimento, comercialização, preparação e consumo de alimentos e bebidas ${ }^{4}$. Na abordagem de sistemas alimentares é necessário considerar todos os determinantes do consumo alimentar a partir das relações estabelecidas entre os diferentes agentes participantes da cadeia: produtores, distribuidores e consumidores ${ }^{5}$.

A alimentação contemporânea tornou-se insustentável $\mathrm{l}^{6,7}$, por ser composta por alimentos que utilizam muita energia para sua produção, que têm grande impacto ambiental e necessitam de vasta extensão de terra para sua produção, podendo exacerbar outros problemas relacionados à produção e ao suprimento de alimentos ${ }^{7}$. Nesse sentido, recomendações para uma alimentação saudável precisam agregar a sustentabilidade como uma de suas principais dimensões. Alimentos saudáveis devem ser relacionados a um sistema alimentar que seja economicamente vi- ável, ambientalmente sustentável e socialmente justo, contemplados por uma alimentação sustentável.

O termo dieta ou alimentação sustentável não é recente, entretanto não apresenta uma definição utilizada amplamente. Foi descrito a primeira vez em 1986 por Gussow e Clancy ${ }^{8}$ como uma dieta composta por alimentos que contribuíssem não somente para a saúde, mas também para a sustentabilidade de todo o sistema alimentar. A complexidade da dieta sustentável foi demonstrada pela Organização das Nações Unidas para agricultura e alimentação (FAO) em 2010. Dietas sustentáveis são definidas como aquelas "com baixo impacto ambiental, que contribuem para a segurança alimentar e nutricional e à vida saudável para as gerações presentes e futuras. Dietas sustentáveis devem proteger e respeitar a biodiversidade e os ecossistemas, culturalmente aceitável e acessível, economicamente justa e acessível; nutricionalmente adequada, segura e saudável; além de otimizar os recursos naturais e humanos"

Apesar da abrangência das dimensões da alimentação sustentável, as pesquisas têm dado enfoque no impacto ambiental, particularmente no clima, em termos de emissão de gases de efeito estufa ${ }^{10}$. Técnicas quantitativas de análise muito usadas, como análise do ciclo de vida ${ }^{11-14}$ possuem enfoque apenas ambiental, ignoram dessa forma, aspectos sociais e econômicos ${ }^{15}$. Partindo dessa premissa, é possível ter uma dieta com baixa emissão de gases de efeito estufa, mas que não seja saudável, ao mesmo tempo em que se pode ter uma dieta saudável e com alta emissão de ga$\mathrm{ses}^{16}$. No entanto, em uma perspectiva ampliada, alimentos com um menor impacto ambiental não são necessariamente mais sustentáveis (em seu sentido amplo) do que outros, se esses outros causarem prejuízos à sociedade. Por exemplo, a eficiência ambiental pode ser alcançada mediante produção em larga escala, mas quando realizada em pequena escala pode apoiar pequenos produtores e contribuir com o desenvolvimento local ${ }^{17}$.

Considerando que não é possível ter uma alimentação saudável sem que seja sustentável em todas as suas dimensões, e a carência dessa reflexão na área de alimentação e nutrição, pretende-se realizar um exercício reflexivo da literatura científica sobre os principais desafios e perspectivas da alimentação saudável e sustentável. Trata-se de uma tentativa de aproximação e problematização da temática, considerando a necessidade de explorar os principais desafios para sua concretização. 


\section{Método}

Visando cumprir o objetivo proposto foi realizada uma revisão narrativa. Os artigos de revisão narrativa são publicações com a finalidade de descrever e discutir o estado da arte de um determinado assunto ${ }^{18}$. Visto a abrangência da temática e a dificuldade em estabelecer uma pergunta de pesquisa precisa, a revisão narrativa foi utilizada por possibilitar uma discussão ampliada.

A revisão foi realizada de forma não sistemática no período de novembro de 2015 a novembro de 2016. As buscas se basearam na pergunta de pesquisa: Quais os desafios para alimentação sustentável, considerando todas as etapas de produção, processamento, comercialização e consumo? A busca bibliográfica foi realizada nas bases de dados Scopus, Pubmed e Google Acadêmico, complementada com uma busca manual nas listas de referências dos trabalhos selecionados. A busca incluiu as palavras-chave sustainable diet ou sustainable nutrition ou sustainable food ou wholesome diet ou wholesome nutrition combinados com production ou processing ou marketing ou consumption. As buscas foram realizadas por um dos autores, sem limitação de data, país do estudo ou área de conhecimento. Foram incluídos no estudo artigos originais, de revisão e literatura cinzenta nos idiomas inglês, espanhol e português. A seleção dos artigos, documentos oficiais nacionais e internacionais abrangeu o período de 1986 a 2016.

As revisões narrativas são consideradas como de menor evidência científica devido à seleção arbitrária de artigos e por estar sujeita a viés de seleção $0^{18,19}$. Contudo, são consideradas essenciais para contribuições no debate de determinadas temáticas, levantando questões e colaborando para a atualização do conhecimento ${ }^{18}$.

Considerando as temáticas relacionadas à pergunta de pesquisa, os resultados foram divididos em quatro seções conforme as etapas do sistema alimentar. Na primeira apresenta-se o contexto e as principais observações sobre a produção de alimentos. Na segunda seção identificam-se os principais desafios relacionados ao processamento de alimentos. Na terceira, a comercialização de alimentos e, por fim, na quarta seção o consumo de alimentos. Nas considerações finais são indicadas algumas perspectivas para o debate sobre alimentação saudável e sustentável.

\section{Resultados e Discussão}

A Figura 1 ilustra os principais contrapontos de sistemas alimentares insustentáveis e sustentáveis observados na revisão de literatura, considerando as etapas percorridas pelo alimento, do campo à mesa do consumidor. A figura resume os pontos considerados relevantes na abordagem da (in) sustentabilidade dos sistemas alimentares e serão discutidos nos itens subsequentes.

A partir dos dados analisados na revisão elaborou-se a Figura 2, uma representação gráfica que sintetiza os principais aspectos relacionados a uma alimentação mais saudável e sustentável, apresentando uma hierarquização de práticas nas etapas de produção, processamento, produção e consumo de alimentos. A questão central de uma alimentação sustentável passa a ser o caminho percorrido pelo alimento que será consumido. Há que se priorizar a diversidade de alimentos como base da alimentação, seguido da definição de uma hierarquia para cada etapa, considerando que os modelos do topo devem ser evitados. Infere-se que um exemplo de alimentação saudável e sustentável está situado na base da figura com: alimentos produzidos seguindo os preceitos agroecológicos, adquiridos frescos diretamente de produtores familiares, para elaboração de refeições culturalmente aceitas.

\section{Produção de alimentos}

Modificações nas formas de produção de alimentos vêm sendo observadas ao longo dos anos. Após a revolução verde, implementada na década de 1950 sob a justificativa de aumentar a produção de alimentos e acabar com a fome, os modos de produção modificaram-se. Iniciou-se uma produção de larga escala com alta tecnologia, dominada por grandes corporações, buscando a maior produtividade ${ }^{20}$. A estrutura da propriedade rural se tornou mais concentrada, aumentaram as disparidades de renda, o êxodo rural e a exploração da força de trabalho nas atividades agrícolas. Tudo isso levou a piora da qualidade de vida da população trabalhadora do campo ${ }^{21}$.

A estrutura fundiária apresenta-se como um dos maiores desafios para a produção sustentável de alimentos no Brasil. Menos de $1 \%$ do número de estabelecimentos agropecuários ocupava cerca de $44 \%$ da área cultivável brasileira em 2006. Grandes áreas no país são destinadas principal- 


\begin{tabular}{|c|c|c|c|c|}
\hline & PRODUÇÃO & PROCESSAMENTO & COMERCIALIZAÇÃO & CONSUMO \\
\hline \multirow{7}{*}{ 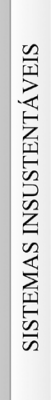 } & Agricultura Convencional & Elevado Processamento & Cadeias longas & Consumo não sustentável \\
\hline & Patronal & Retirada de nutrientes & $\begin{array}{l}\text { Grande número de } \\
\text { intermediários }\end{array}$ & Hábitos não saudáveis \\
\hline & Monocultura & Refinamento & Longas distâncias & $\begin{array}{l}\text { Indisposição para comprar } \\
\text { produtos sustentáveis }\end{array}$ \\
\hline & Transgênicos & Adição de gordura trans & $\begin{array}{c}\text { Desvalorização do produtos } \\
\text { locais }\end{array}$ & $\begin{array}{l}\text { Elevado consumo de alimentos } \\
\text { ultraprocessados }\end{array}$ \\
\hline & Agrotóxicos & $\begin{array}{l}\text { Adição de aditivos e } \\
\text { conservantes }\end{array}$ & Preços elevados & $\begin{array}{l}\text { Busca por alimentos de rápido } \\
\text { preparo }\end{array}$ \\
\hline & Criação intensiva de animais & $\begin{array}{c}\text { Aditivos baseados em } \\
\text { subprodutos de soja e milho }\end{array}$ & $\begin{array}{l}\text { Valorização de grandes redes } \\
\text { varejistas }\end{array}$ & Alimentação não diversificada \\
\hline & \multicolumn{4}{|c|}{ Elevado desperdício: alimentos, energia, água } \\
\hline \multirow{7}{*}{ 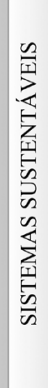 } & Agroecologia & Baixo Processamento & Cadeias curtas & Consumo sustentável \\
\hline & Agricultura familiar & Manutenção dos nutrientes & $\begin{array}{c}\text { Nenhum ou pequeno número de } \\
\text { intermediários }\end{array}$ & $\begin{array}{l}\text { Alimentos frescos, } \\
\text { agroecológicos }\end{array}$ \\
\hline & Diversificada & Processamento mínimo & $\begin{array}{l}\text { Proximidade do produtor e do } \\
\text { consumidor }\end{array}$ & $\begin{array}{l}\text { Disponibilidade para comprar } \\
\text { produtos sustentáveis }\end{array}$ \\
\hline & Orgânicos & Sem adição de gordura trans & $\begin{array}{c}\text { Comércio justo e economia } \\
\text { solidária }\end{array}$ & $\begin{array}{c}\text { Compra direta de agricultores } \\
\text { familiares }\end{array}$ \\
\hline & Sazonais & Sem adição de conservantes & $\begin{array}{l}\text { Valorização do produto e do } \\
\text { produtor }\end{array}$ & $\begin{array}{c}\text { Alimentos regionais, } \\
\text { tradicionais, diversificados }\end{array}$ \\
\hline & $\begin{array}{l}\text { Integração lavoura-pecuária- } \\
\text { floresta }\end{array}$ & Sem outros aditivos alimentares & Confiança no produtor & Habilidades culinárias \\
\hline & \multicolumn{4}{|c|}{ Baixo desperdício: alimentos, energia, água } \\
\hline
\end{tabular}

Figura 1. Principais contrapontos de sistemas alimentares insustentáveis e sustentáveis observados na revisão de literatura.

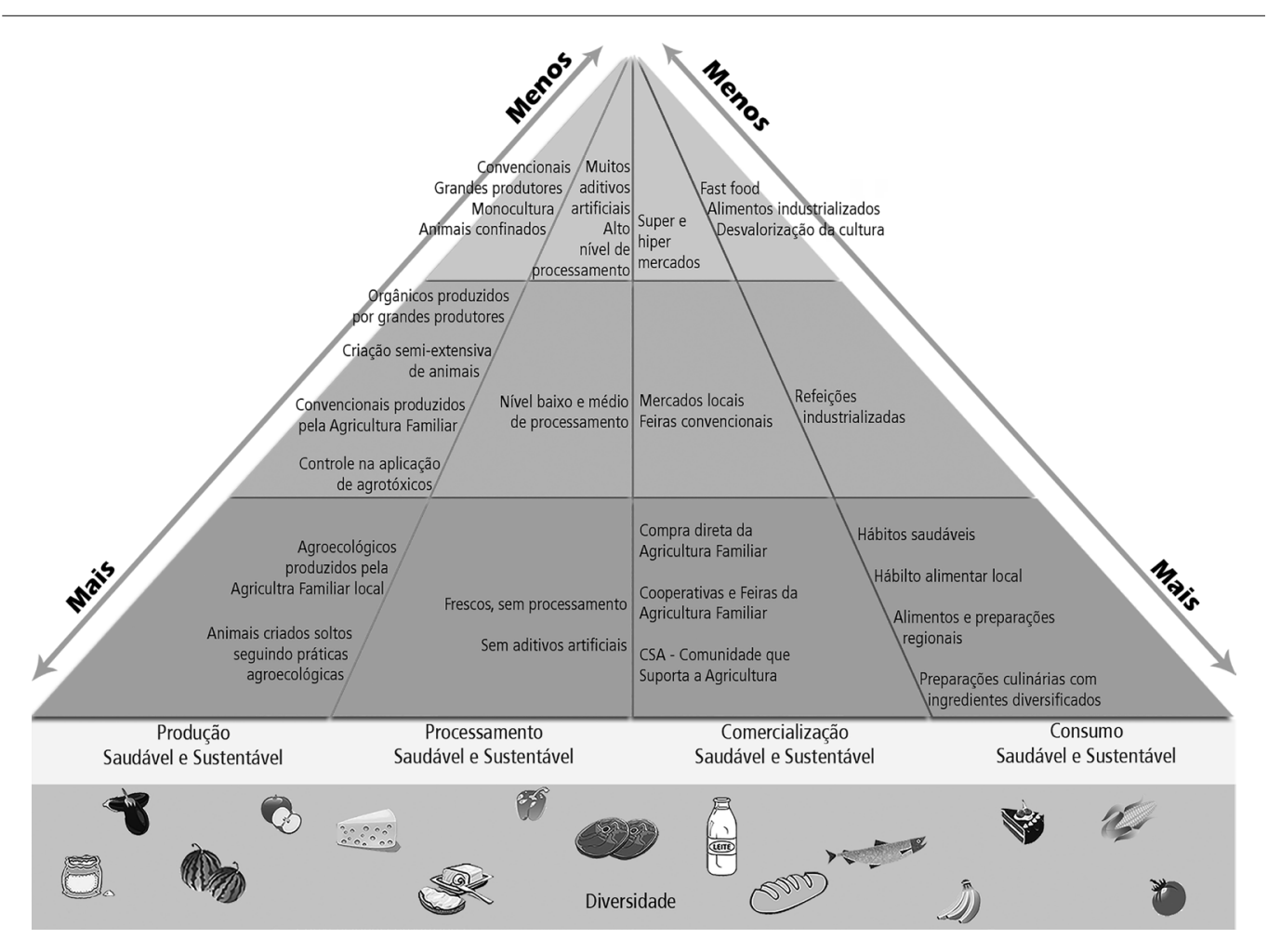

Figura 2. Representação gráfica de orientação e operacionalização para uma alimentação mais saudável e sustentável. 
mente para a criação bovina e monocultura de soja, milho e algodão, o que vem aumentando a desigualdade que caracteriza a propriedade da terra no Brasil ${ }^{22}$.

A criação animal está associada com elevado impacto ambiental, contribuindo para as alterações climáticas, degradação do solo, emissão de gases, contaminação da água e perda da biodiversidade ${ }^{23,24}$. Sugere-se que a adoção de uma dieta sem produtos animais poderia reduzir até $50 \%$ da emissão de gases e uso da terra ${ }^{25}$. Algumas medidas podem ser adotadas para atenuar a agressão ambiental causada pela criação animal. O processo de integração dos animais com produção de alimentos e floresta é apontado como uma possibilidade sustentável para a produção ${ }^{26}$. Também são necessárias políticas internacionais para a proteção ambiental na produção de peixes e frutos do mar e sistemas de certificação para as empresas $^{27}$. Apesar das possibilidades de um sistema produtivo mais sustentável, a redução do consumo de carnes e derivados pela população é apontada como emergencial, visto seu elevado consumo em todo o mundo ${ }^{24}$. Contudo, é importante discutir a forma de produção nesse contexto, já que uma dieta onívora baseada em produtos orgânicos pode ter menor impacto ambiental que uma dieta vegetariana composta por alimentos produzidos com elevado uso de agrotóxicos ${ }^{28}$.

Além das preocupações com a liberação de gases prejudiciais ao ambiente, a criação animal nos sistemas intensivos gera grande discussão pelo fato da alimentação ser baseada em cereais como o milho e a soja, o que exige grandes áreas para o cultivo. A produção de soja e milho vem aumentando nos últimos anos, especialmente com sementes transgênicas. Uma das justificativas iniciais para a utilização de alimentos transgênicos era a redução do uso de agrotóxicos para o plantio. No entanto, a inserção de genes resistentes aos agrotóxicos em alguns cultivos confere resistência às pragas e às ervas daninhas, desequilibrando os ecossistemas, implicando no uso de uma maior quantidade de agrotóxicos ${ }^{29}$. A produção de transgênicos apresenta alto risco à sustentabilidade do sistema alimentar principalmente pela perda da biodiversidade, pelo uso de agrotóxicos e pela contaminação de sementes crioulas $^{30,31}$.

O Brasil ocupa a primeira posição no uso de agrotóxicos em todo o mundo desde 2008, influenciado pela produção de soja ${ }^{31}$. O consumo de alimentos transgênicos com agrotóxicos associados tem sido relacionado a problemas neuro- lógicos, alterações hormonais, infertilidade, cân$\operatorname{cer}^{32}$ e doença celíaca em humanos ${ }^{33}$. A exposição ocupacional a agrotóxicos está relacionada, entre outras doenças, a maior incidência de cânceres nos diferentes órgãos e sistemas ${ }^{34}$, problemas respiratórios $^{35}$ e doenças crônicas ${ }^{36}$. Além dos agricultores, a exposição da população no entorno também apresenta relação com doenças, como atrasos no desenvolvimento mental e ocorrência de desordens mentais em crianças ${ }^{37}$ e maior ocorrência de malformações congênitas em bebês devido a exposição materna a agrotóxicos ${ }^{38}$.

Para a nutrição, um importante embasamento para o consumo de alimentos orgânicos é a superioridade da qualidade nutricional comparado aos alimentos convencionais. Alimentos produzidos de maneira orgânica contêm maior nível absoluto e conteúdo total de micronutrientes $^{39}$, maior teor de polifenóis, ácidos fenólicos, isoflavonas, estilbeno e antocianinas ${ }^{40}$. Estudos também identificaram maiores teores de ácidos graxos poliinsaturados totais e ácidos graxos poli-insaturados ômega 3 em carnes orgânicas ${ }^{41}$ e maiores concentrações de ácido alfa-linolênico, total de ácidos graxos ômega-3, proteína, gordura, ácidos graxos poli-insaturados e ácido eicosapentanoico em produtos lácteos orgânicos quando comparados ao convencional ${ }^{42}$. Além disso, o nível de cádmio e metal pesado tóxico nos alimentos convencionais é duas vezes maior que nos alimentos orgânicos ${ }^{40}$. Pesquisadores sugerem que o consumo de alimentos orgânicos aumentaria de 20 a $40 \%$ a ingestão de antioxidantes ${ }^{40}$.

Em relação ao impacto ambiental, o sistema de produção orgânico contribui para a manutenção de maior matéria orgânica no solo, menores perdas de nutrientes, menor uso de energia e maior biodiversidade ${ }^{43}$. Nesse sentido, a agroecologia aparece como um novo caminho para a agricultura e, consequentemente, para uma alimentação saudável e sustentável. A agricultura familiar é considerada ideal para abrigar o desenvolvimento de uma agricultura ambientalmente sustentável, em função de suas características de produção diversificada, integrando atividades vegetais e animais, e por trabalhar em menores escalas $^{44}$. Mais de 12 milhões de pessoas estão vinculadas à agricultura familiar e são responsáveis pela produção da maioria dos alimentos básicos como arroz, feijão, mandioca, batata e vários tipos de legumes, verduras e frutas. O consumo desses alimentos tem como consequência natural o estímulo da agricultura familiar e da economia local $^{45}$. 


\section{Processamento}

O processamento de alimentos refere-se a métodos empregados pelos fabricantes visando transformar produtos primários ${ }^{46}$. Esse processo pode ser benéfico sob vários aspectos: aumentar a variedade de alimentos consumidos, possibilitar a preservação, o armazenamento por períodos maiores, a segurança, a palatabilidade e a conveniência ${ }^{47}$. A aplicação de níveis elevados de processamento com aplicação de técnicas de conservação pode representar um elevado risco para a alimentação saudável e sustentável, particularmente quando se perde grande parte dos nutrientes do alimento em sua composição original e adicionam-se gorduras, sódio, açúcares, aditivos e conservantes.

O processamento para a fabricação de farinhas é um exemplo para essa questão. O grão em sua composição original é processado e a fração refinada pode perder $90 \%$ das vitaminas e minerais ${ }^{48}$. Alimentos com alto grau de processamento e conservação são os maiores contribuintes para o elevado consumo de gorduras em geral, de gordura saturada, de gordura trans e de açúcar livre; e menor teor de fibras, de proteínas e de potássio na dieta de brasileiros ${ }^{49}$. O consumo desses alimentos está relacionado a um processo que pode dificultar a percepção da origem dos ingredientes que compõem um determinado alimento, distanciando as pessoas do ato cultural de se alimentar ${ }^{50}$.

O processamento excessivo também não é sustentável em outras dimensões, como a social. Esse tipo de atividade geralmente é realizada por indústrias de grande porte ${ }^{45}$ e não por pequenos produtores, os produtos produzidos são comercializados por grandes redes de supermercado e não por cadeias curtas que beneficiam os pequenos produtores locais. Não existe valor para o agricultor, para a manutenção da agricultura e para a diversidade biológica quando os alimentos são convertidos em versões refinadas com alto conteúdo de sal e açúcar ${ }^{8}$. Além disso, a inserção de grandes empresas no setor alimentício possibilita a comercialização desses alimentos com um preço muito reduzido, consumidos largamente pela população ${ }^{51}$.

Nesse sentido, o Guia Alimentar para a População Brasileira recomenda que se evitem produtos processados produzidos por grandes indústrias, particularmente pela composição nutricional e pelo impacto que suas formas de produção, distribuição, comercialização e consumo têm sobre a cultura, a vida social e sobre o ambiente ${ }^{45}$. Para além de a população evitar esses produtos, existe a necessidade de desenvolvimento de políticas públicas que incentivem e facilitem as escolhas saudáveis, já que mudanças estruturais no sistema agroalimentar brasileiro não acontecerão por iniciativas espontâneas do setor produtivo ${ }^{52}$. Políticas alimentares que promovam alimentação equilibrada têm o potencial de contribuir para o desenvolvimento sustentável ${ }^{53}$. No âmbito da industrialização dos alimentos, são necessárias regulamentações para o processamento, rotulagem e publicidade, além de fiscalização para o cumprimento das obrigações. Contudo, ainda existem muitas tensões que envolvem as negociações entre representantes da indústria de alimentos e o Estado ${ }^{54}$, o que dificulta o fornecimento de alimentos saudáveis e sustentáveis à população.

\section{Comercialização}

A entrada de grandes corporações internacionais no varejo gerou uma dinâmica diferente da observada pela comercialização em pequenos mercados, determinada por agentes concentradores, o que resulta na exclusão de alguns ${ }^{55}$. Estamos situados em um processo hegemônico e com muitas barreiras, contudo, experiências exitosas sobre a valorização dos modos de produção artesanais e de comércio justo demonstram a possibilidade de modificação e benefícios para produtores e consumidores ${ }^{56}$.

Sistemas alimentares locais e regionais têm o potencial de promover sistemas alimentares sustentáveis ${ }^{57}$. O conceito de sistemas alimentares localizados é discutido e tem diferentes interpretações. De maneira abrangente, busca-se integrar as etapas de produção, transformação, distribuição e consumo visando melhorar a economia, o ambiente e a saúde de um lugar específico e a construção de economias alimentares autossustentadas e mais baseadas no local ${ }^{58}$. Lang relata a importância do consumo local para reintegrar hábitos alimentares antigos, para valorizar o alimento e os produtores da região $0^{59}$.

Ao discutir a comercialização de alimentos deve-se priorizar inicialmente os circuitos curtos de comercialização, caracterizado pelo reduzido número de intermediários e a proximidade geográfica $^{60}$. No Brasil, intervenções governamentais demonstram as potencialidades do Estado na reorganização do sistema agroalimentar e das relações de mercado a partir do foco no desenvolvimento rural sustentável ${ }^{61}$. O Programa Nacional de Alimentação Escolar (PNAE) e o Programa de 
Aquisição de Alimentos (PAA), particularmente a modalidade Compra institucional, já demonstraram que é possível realizar compra local de alimentos, e assim beneficiar produtores e consumidores $^{61}$. Nesse sentido, em um sistema dominado pelas trocas capitalistas, as políticas públicas vêm introduzindo uma dimensão de reciprocidade e de justiça às relações ${ }^{62}$. Essas relações de reciprocidade reduzem os custos de produção ou de transação e, apesar da concorrência do sistema capitalista, geralmente desfavorável para seus sistemas de produção, permitem o acesso dos agricultores familiares ou camponeses aos mercados, principalmente institucionais ${ }^{62}$. Isto geralmente se dá por meio da criação de redes, de associações produtivas de redes agroalimentares alternativa ${ }^{56}$ e programas governamentais.

Em nível individual, a compra direta de agricultores familiares aparece como estratégia de aproximação da produção. Os mercados públicos com preços garantidos constituem um instrumento de política pública de interface entre sistemas de troca mercantil e sistemas de reciprocidade $^{62}$. Ainda, circuitos curtos de comercialização podem ser observados em vendas diretas na propriedade, entregas de cestas em domicílio, lojas especializadas, cooperativas e restaurantes que compram direto do produtor ${ }^{60}$, e também em hortas comunitárias, agricultura urbana e a iniciativa "Comunidade que Sustenta a Agricultura" (CSA) ${ }^{63}$. Essas formas de conexão da produção com o consumo aproximam o consumidor da origem do alimento. Autores perceberam que essa aproximação é benéfica e está relacionada a um padrão diferenciado na percepção da alimentação saudável, ampliando o conceito para questões culturais e sustentáveis ${ }^{63}$.

\section{Consumo sustentável}

O consumo de alimentos vem sofrendo alterações que provocam impactos negativos para a saúde e para o ambiente. A alimentação atual baseada em produtos com alto valor energético e pobres em diversidade apoia e incentiva a intensificação da agricultura, além de agravar a tendência para uma epidemia de obesidade global ${ }^{64}$. O consumo de alimentos em excesso também é tratado por autores como contrário à sustentabilidade ${ }^{8,65}$, já que ultrapassa a necessidade do indivíduo, tornando-se desperdício.

O patrimônio cultural, a qualidade dos alimentos e as habilidades culinárias são considerados aspectos-chave para os padrões alimentares sustentáveis e a segurança alimentar ${ }^{66}$. Contudo, alterações nos padrões e escolhas alimentares da população apresentam-se como um grande desafio da alimentação sustentável. As transformações sociais, econômicas e culturais ocorridas na sociedade representam grande impacto na alimentação ${ }^{55}$, sendo necessário considerar nessa abordagem os estilos de vida modernos, expectativas culturais e sociais e o ambiente em que são feitas as escolhas alimentares ${ }^{16}$.

O Brasil detém de 15 a 20\% da biodiversidade mundial ${ }^{67}$, contudo, observa-se uma redução real das variedades alimentares consumidas, o que pode comprometer a garantia da segurança alimentar e nutricional e a soberania alimentar ${ }^{68}$. Apesar de se conhecerem mais de sete mil espécies de plantas comestíveis, apenas 30 culturas fornecem $95 \%$ da ingestão diária de calorias para a população ${ }^{69}$. A conservação da diversidade de espécies vegetais comestíveis é considerada chave para o abastecimento de alimentos ${ }^{70}$. Nesse sentido, surge o estímulo ao consumo de Plantas Alimentícias Não Convencionais (PANC) e alimentos da sociobiodiversidade, que se inseridas na alimentação cotidiana, podem aumentar a variedade e a qualidade da alimentação, já que a qualidade nutricional dessas plantas é superior àquelas domesticadas ${ }^{71}$. Essas plantas apresentam grande potencial para aumentar a diversidade da dieta, proporcionando melhoria da qualidade nutricional, sendo acessível para populações em situação de vulnerabilidade social ${ }^{72}$.

Mudanças individuais na dieta podem ter grande potencial para influenciar a demanda por certos alimentos e diminuir a pressão sobre o sistema alimentar global. Para isso, é preciso instrumentalizar os consumidores e influenciar no comportamento de escolha ${ }^{73}$. A divulgação de informações para a população é uma das metas dos países signatários dos Objetivos do Desenvolvimento Sustentável da ONU até $2030^{74}$. Por isso enfatiza-se a necessidade das diretrizes dietéticas incorporarem recomendações diretas de sustentabilidade $e^{8,75}$.

Para isso, será necessário considerar estilos de vida modernos, expectativas culturais e sociais e o ambiente em que são feitas as escolhas alimentares $^{76}$. Uma rotulagem de alimentos que possa auxiliar na escolha de uma dieta saudável e sustentável é necessária ${ }^{77}$. É preciso também que a população se informe sobre os alimentos comprados nos supermercados e consumidos nos restaurantes, bem como visite as propriedades rurais ${ }^{78}$. O movimento Slow Food destaca o papel político do consumidor em suas escolhas ao chamá-lo de "co-produtor", ou seja, responsá- 
vel também pela forma com que os alimentos são produzidos $^{79}$.

No sentido de dar orientações à população, o Guia Alimentar para a População Brasileira vem, desde 2006, abordando questões sobre alimentação sustentável. Segundo o Guia, a alimentação saudável deve ser incentivada pelo consumo de alimentos nas formas mais naturais, produzidos localmente e pela agricultura familiar, que valorizem os alimentos regionais e a cultura alimentar. Além disso, deve estimular o resgate aos bons hábitos alimentares. Reconhece como prioritária a produção de alimentos que fomentem e garantam a SAN, com o uso da terra e da água de forma ecologicamente sustentável e com impactos sociais e ambientais positivos ${ }^{46}$. O Guia Alimentar brasileiro lançado em $2014^{45}$, relaciona a alimentação saudável com a perspectiva de aproximar produção e consumo, por estímulo da aquisição em feiras e outros mercados institucionais, nas práticas alimentares de base agroecológica, e no resgate dos saberes tradicionais de produção e processamento de alimentos pela agricultura familiar ${ }^{80}$. É reconhecido como um dos guias alimentares que mais insere recomendações de sustentabilidade em suas orientações dietéticas ${ }^{81}$. Ressalta-se que orientações para alimentação saudável afetam a saúde da população, mas apresentam também potencial impacto na produção agrícola, comércio e economia ${ }^{82}$.

\section{Considerações finais}

A resposta à pergunta "Quais as recomendações para uma alimentação saudável e sustentável?” ainda não é consenso na literatura. Exige uma abordagem holística baseada em evidências para que a discussão seja aprofundada e traga benefícios para a sociedade, para a economia e para o ambiente. Contudo, reconhece-se que existe um grande desafio para compreender a interação entre todos os indicadores de uma dieta sustentável nos diferentes contextos socioeconômicos e ambientais ${ }^{82}$.

Para que seja possível a concretização de um sistema mais sustentável, é necessário que a po- pulação tenha acesso a distintas formas de produção, processamento e comercialização. Dessa forma, salienta-se a intervenção do Estado nas políticas alimentares como fator essencial para a consolidação da alimentação sustentável. Na perspectiva do consumo, considera-se fundamental a indissociabilidade entre a promoção da alimentação saudável e sustentável e que as premissas de ambas sejam articuladas e consideradas em recomendações alimentares.

É importante destacar que não se teve a pretensão de abordar nesse artigo todos os possíveis aspectos do campo da alimentação e sustentabilidade, mas sim iniciar uma aproximação e um exercício reflexivo. A abordagem de uma alimentação saudável e sustentável deve considerar interações multidimensionais em todo o sistema alimentar, bem como a necessidade de maior reflexão e engajamento envolvendo diversas áreas e representantes de todas as etapas do sistema alimentar.

\section{Colaboradores}

SS Martinelli participou da concepção, estruturação e redação do trabalho; SB Cavalli participou da concepção, estruturação e revisão crítica do trabalho. Ambos os autores aprovaram a versão final encaminhada.

\section{Agradecimentos}

Os autores agradecem a Fundação de Amparo à Pesquisa e Inovação do Estado de Santa Catarina (FAPESC), pela bolsa de estudos de Doutorado concedida a SSM. 


\section{Referências}

1. Escudero P. Alimentación. Buenos Aires: Hachette; 1934.

2. World Health Organization (WHO). Noncommunicable diseases progress monitor, 2015. Geneva: WHO; 2015.

3. World Health Organization (WHO). Global Strategy on Diet, Physical Activity and Health. Geneva: WHO; 2004. [Eighth plenary meeting, Committee A, third report].

4. Sobal J, Kettel Khan L, Bisogni C. A conceptual model of the food and nutrition system. Soc Sci Med 1998; 47(7):853-863.

5. Oliveira SP, Thébaud-Mony A. Estudo do consumo alimentar: em busca de uma abordagem multidisciplinar. Rev Saude Publica 1997; 31(2):201-208.

6. Auestad N, Fulgoni VL, III. What current literature tells us about sustainable diets: Emerging research linking dietary patterns, environmental sustainability, and economics. Advances in Nutrition 2015; 6(1):19-36.

7. Gliessman SR. Agroecologia: processos ecológicos em agricultura sustentável. Porto Alegre: Ed. Univ. Federal do Rio Grande do Sul; 2001.

8. Gussow JD, Clancy KL. Dietary guidelines for sustainability. J Nutr Educ 1986; 18(1):1-5.

9. Burlingame BA, Dernini S. Sustainable diets and biodiversity. In: Food and Agriculture Organization of the United Nations, editor. International Scientific Symposium Biodiversity and Sustainable Diets United against Hunger (2010: Rome, Italy). Rome: International Scientific Symposium Biodiversity and Sustainable Diets United against Hunger (2010: Rome, Italy); 2012.

10. Nordic Council of Ministers. Nordic Nutrition Recommendations 2012: Integrating nutrition and physical activity. Copenhagen: Nordic Council of Ministers; 2014.

11. Calderón LA, Iglesias L, Laca A, Herrero M, Díaz M. The utility of Life Cycle Assessment in the ready meal food industry. Resour Conserv Recycl 2010; 54(12):1196-1207.

12. Del Borghi A, Gallo M, Strazza C, Del Borghi M. An evaluation of environmental sustainability in the food industry through Life Cycle Assessment: the case study of tomato products supply chain. J Clean Prod 2014; 78:121-130.

13. Cerutti AK, Contu S, Ardente F, Donno D, Beccaro GL. Carbon footprint in green public procurement: Policy evaluation from a case study in the food sector. Food Policy 2016; 58:82-93.

14. Baldwin C, Wilberforce N, Kapur A. Restaurant and food service life cycle assessment and development of a sustainability standard. Int J Life Cycle Assess 2011; 16(1):40-49.

15. Morgan K. Greening the realm: sustainable food chains and the public plate. Regional Studies 2008; 42(9):1237-1250.

16. Macdiarmid JI, Kyle J, Horgan GW, Loe J, Fyfe C, Johnstone A, McNeill G. Sustainable diets for the future: can we contribute to reducing greenhouse gas emissions by eating a healthy diet? Am J Clin Nutr 2012; 96(3):632-639.

17. Goggins $\mathrm{G}$, Rau H. Beyond calorie counting: assessing the sustainability of food provided for public consumption. J Clean Prod 2016; 112(1):257-266.
18. Rother ET. Revisão sistemática $\mathrm{X}$ revisão narrativa Acta Paul Enferm 2007; 20:v-vi.

19. Cordeiro AM, Oliveira GM, Rentería JM, Guimarães CA. Revisão sistemática: uma revisão narrativa. Rev Col Bras Cir 2007; 34:428-431.

20. Cavalli SB. Segurança alimentar: a abordagem dos alimentos trangênicos. Rev Nutr 2001; 14(Supl.):41-46.

21. Palmeira M. Modernização, Estado e questão agrária. Estudos Avançados 1989; 3:87-108.

22. Instituto Brasileiro de Geografia e Estatística (IBGE). Censo Agropecuário. Brasil, grandes regiões e unidades da Federação. Rio de Janeiro: IBGE; 2009.

23. Ruviaro CF, Costa JS, Florindo TJ, Rodrigues W, Medeiros GIB, Vasconcelos PS. Economic and environmental feasibility of beef production in different feed management systems in the Pampa biome, southern Brazil. Ecological Indicators 2016; 60:930-939.

24. Machovina B, Feeley KJ, Ripple WJ. Biodiversity conservation: The key is reducing meat consumption. Sci Total Environ 2015; 536:419-431.

25. Hallström E, Carlsson-Kanyama A, Börjesson P. Environmental impact of dietary change: a systematic review. J Clean Prod 2015; 91:1-11.

26. Balbino LC, Barcellos AO, Stone LF. Marco referencial integração lavoura-pecuária-floresta. Brasília: Embrapa; 2011.

27. Bostock J, McAndrew B, Richards R, Jauncey K, Telfer T, Lorenzen K, Little D, Ross L, Handisyde N, Gatward I, Corner R. Aquaculture: global status and trends. Philos Trans R Soc Lond B Biol Sci 2010; 365(1554):2897-2912.

28. Baroni L, Cenci L, Tettamanti M, Berati M. Evaluating the environmental impact of various dietary patterns combined with different food production systems. Eur J Clin Nutr 2006; 61(2):279-286.

29. Bonny S. Genetically Modified Herbicide-Tolerant Crops, Weeds, and Herbicides: Overview and Impact. J Environ Manage 2016; 57(1):31-48.

30. Nodari RO, Guerra MP. Avaliação de riscos ambientais de plantas transgênicas. Cadernos de Ciência \& Tecnologia 2001; 18(1):81-116.

31. Carneiro FF, Augusto LGS, Rigotto RM, Friedrich K, Búrigo AC. Dossiê ABRASCO: um alerta sobre os impactos dos agrotóxicos na saúde. Rio de Janeiro: EPSJV; 2015.

32. Friedrich K. Parecer técnico: Avaliação dos efeitos tóxicos sobre o sistema reprodutivo, hormonal e câncer para seres humanos após o uso do herbicida 2,4-D. Rio de Janeiro: Fiocruz; 2014.

33. Samsel A, Seneff S. Glyphosate, pathways to modern diseases II: Celiac sprue and gluten intolerance. Interdiscip Toxicol 2013; 6(4):159-184.

34. Alavanja MCR, Bonner MR. Occupational pesticide exposures and cancer risk: A review. J toxicol environ health 2012; 15(4):238-263.

35. Faria NMX, Facchini LA, Fassa AG, Tomasi E. Pesticides and respiratory symptoms among farmers. Rev Saude Publica 2005; 39(6):973-981.

36. Gangemi S, Miozzi E, Teodoro M, Briguglio G, De Luca A, Alibrando C, Polito I, Libra M. Occupational exposure to pesticides as a possible risk factor for the development of chronic diseases in humans (Review). Mol Med Rep 2016; 14(5):4475-4488. 
37. Roberts JR, Karr CJ, Paulson JA, Brock-Utne AC, Brumberg HL, Campbell CC, Lanphear BP, Osterhoudt KC, Sandel MT, Trasande L, Wright RO. Pesticide exposure in children. Pediatrics 2012; 130(6):e1765-e88.

38. Oliveira NP, Moi GP, Atanaka-Santos M, Silva AMC, Pignati WA. Malformações congênitas em municípios de grande utilização de agrotóxicos em Mato Grosso, Brasil. Cien Saude Colet 2014; 19(10):4123-4130.

39. Hunter D, Foster M, McArthur JO, Ojha R, Petocz P, Samman S. Evaluation of the micronutrient composition of plant foods produced by organic and conventional agricultural methods. Crit Rev Food Sci Nutr 2011; 51(6):571-582.

40. Barański M, Średnicka-Tober D, Volakakis N, Seal C, Sanderson R, Stewart GB, Benbrook C, Biavati B, Markellou E, Giotis C, Gromadzka-Ostrowska J, Rembiałkowska E, Skwarło-Sońta K, Tahvonen R, Janovská D, Niggli U, Nicot P, Leifert C. Higher antioxidant and lower cadmium concentrations and lower incidence of pesticide residues in organically grown crops: a systematic literature review and meta-analyses. $\mathrm{Br} J \mathrm{Nutr}$ 2014; 112(5):794-811.

41. Srednicka-Tober D, Baranski M, Seal C, Sanderson R, Benbrook C, Steinshamn H, Gromadzka-Ostrowska J, Rembiałkowska E, Skwarło-Sońta K, Eyre M, Cozzi G, Krogh Larsen M, Jordon T, Niggli U, Sakowski T, Calder PC, Burdge GC, Sotiraki S, Stefanakis A, Yolcu $\mathrm{H}$, Stergiadis S, Chatzidimitriou E, Butler G, Stewart G, Leifert C. Composition differences between organic and conventional meat: a systematic literature review and meta-analysis. Br J Nutr 2016; 115(6):9941011.

42. Palupi E, Jayanegara A, Ploeger A, Kahl J. Comparison of nutritional quality between conventional and organic dairy products: A meta-analysis. J Sci Food Agric 2012; 92(14):2774-2781.

43. Tuomisto HL, Hodge ID, Riordan P, Macdonald DW. Does organic farming reduce environmental impacts? - A meta-analysis of European research. J Environ Manage 2012; 112:309-320.

44. Carmo MS. A produção familiar como locus ideal da agricultura sustentável. Agricultura em São Paulo 1998; 45(1):1-15.

45. Brasil. Ministério da Saúde (MS). Guia Alimentar para a população brasileira. 2a ed. Brasília: MS; 2014.

46. Brasil. Ministério da Saúde (MS). Guia alimentar para a população brasileira: promovendo a alimentação saudável. Brasília: MS; 2006.

47. Floros JD, Newsome R, Fisher W, Barbosa-Cánovas GV, Chen H, Dunne CP, German JB, Hall RL, Heldman DR, Karwe MV, Knabel SJ, Labuza TP, Lund D, Newell-McGloughlin M, Robinson JL, Sebranek JG, Shewfelt RL, Tracy WF, Weaver CM, Ziegler GR. Feeding the World Today and Tomorrow: The Importance of Food Science and Technology. Compr Rev Food Sci Food Saf 2010; 9(5):572-599.

48. Oghbaei M, Prakash J. Effect of primary processing of cereals and legumes on its nutritional quality: A comprehensive review. Cogent Food \& Agriculture 2016; 2(1):1136015.

49. Louzada MLC, Baraldi LG, Steele EM, Martins APB, Canella DS, Moubarac JC, Levy RB, Cannon G, Afshin A, Imamura F, Mozaffarian D, Monteiro CA. Consumption of ultra-processed foods and obesity in Brazilian adolescents and adults. Prev Med 2015; 81:9-15.
50. Proença RPC. Alimentação e globalização: algumas reflexões. Cie Cult 2010; 62:43-47.

51. Monteiro CA, Cannon G. The Impact of Transnational "Big Food" Companies on the South: A View from Brazil. PLOS Medicine 2012; 9(7):e1001252.

52. Castro IRR. Challenges and perspectives for the promotion of adequate and healthy food in Brazil. Cad Saude Publica 2015; 31(1):7-9.

53. Schirnding YV, Yach D. Unhealthy consumption threatens sustainable development. Rev Saude Publica 2002; 36(4):379-382.

54. Henriques P, Dias PC, Burlandy L. A regulamentação da propaganda de alimentos no Brasil: convergências e conflitos de interesses. Cad Saude Publica 2014; 30(6):1219-1228.

55. Fonseca AB, Souza TSNd, Frozi DS, Pereira RA. Modernidade alimentar e consumo de alimentos: contribuições sócio-antropológicas para a pesquisa em nutrição. Cien Saude Colet 2011; 16(9):3853-3862.

56. Wilkinson J. A pequena produção e sua relação com os sistemas de distribuição. In: Wilkinson J, editor. Mercados, redes e valores: O novo mundo da agricultura familiar. Porto Alegre: EDUFRGS; 2008. p. 125-150.

57. Morley A, McEntee J, Marsden T. Food futures: Framing the crisis. In: Marsden T, Morley A, editors. Sustainable food systems: building a new paradigm. Abingdon: Routledge; 2014. p. 221.

58. Feenstra G. Creating space for sustainable food systems: Lessons from the field. Agric Human Values 2002; 19(2):99-106.

59. Lang T. Sustainable diets and biodiversity: The challenge for policy, evidence and behaviour change. In: Burlingame B, Dernini S, editors. Sustainable Diets and Biodiversity. Rome: FAO; 2012. p. 20-26.

60. Darolt MR, Lamine C, Brandemburg A. A diversidade dos circuitos curtos de alimentos ecológicos: ensinamentos do caso brasileiro e francês. Agriculturas 2013; 10(2):5.

61. Food and Agriculture Organization of the United Nations (FAO). Superação da fome e da probreza rural Iniciativas brasileiras. Brasília: FAO; 2016.

62. Sabourin E. Acesso aos mercados para a agricultura familiar: uma leitura pela reciprocidade e a economia solidária. Rev Econ NE 2014; 45:21-35.

63. O'Kane G. A moveable feast: Contemporary relational food cultures emerging from local food networks. Appetite 2016; 105:218-231.

64. Etiévant P. Dietary behaviours and practices: Determinants, action, outcomes. In: Burlingame B, Dernini $\mathrm{S}$, editors. Sustainable diets and biodiversity. Rome: FAO; 2012. p. 102-107.

65. Macdiarmid JI. Is a healthy diet an environmentally sustainable diet? The Proc Nutr Soc 2013; 72(01):1320.

66. Lairon D. Biodiversity and sustainable nutrition with a food-based approach. In: Burlingame B, Dernini S, editor. Sustainable Diets and Biodiversity. Rome: FAO; 2012. p. 30-35.

67. Coradin L, Siminski A, Reis A. Espécies Nativas da Flora Brasileira de Valor Econômico Atual ou Potencial. Brasília: Ministério do Meio Ambiente; 2011.

68. Allen T, Prosperi P, Cogill B, Flichman G. Agricultural biodiversity, social-ecological systems and sustainable diets. Proc Nutr Soc 2014; 73(4):498-508. 
69. Food and Agriculture Organization of the United Nations (FAO). Biodiversity: for a world without hunger. FAO [cited 2017 Nov 10]. [Available from: http:// www.fao.org/biodiversity/components/plants/en/

70. Prescott-Allen R, Prescott-Allen C. How Many Plants Feed the World? Conservation Biology 1990; 4(4):365374.

71. Kinupp VF, Barros IBI. Teores de proteína e minerais de espécies nativas, potenciais hortaliças e frutas. Ciênc Tecnol Aliment 2008; 28(4):846-857.

72. Termote C, Raneri J, Deptford A, Cogill B. Assessing the potential of wild foods to reduce the cost of a nutritionally adequate diet: An example from eastern Baringo District, Kenya. Food Nutr Bull 2014; 35(4):458-479.

73. Riley H, Buttriss JL. A UK public health perspective: what is a healthy sustainable diet? Nutr Bull 2011; 36(4):426-431.

74. Organização das Nações Unidas (ONU). Transformando Nosso Mundo: a Agenda 2030 para o Desenvolvimento Sustentável. Nova York: ONU; 2015.

75. Horgan GW, Perrin A, Whybrow S, Macdiarmid JI. Achieving dietary recommendations and reducing greenhouse gas emissions: modelling diets to minimise the change from current intakes. Int J Behav Nutr Phys Act 2016; 13(1):46.

76. Macdiarmid JI, Kyle J, Horgan GW, Loe J, Fyfe C, Johnstone A, McNeill G. Sustainable diets for the future: can we contribute to reducing greenhouse gas emissions by eating a healthy diet? Am J Clin Nutr 2012; 96(3):632-639.

77. Public Health Association of Australia (PHAA). Ecologically Sustainable Diets. Canberra: PHAA; 2015.

78. Sustainable Table. What you can do. Sustainable Table [cited 2017 Oct 7]. Available from: https://sustainabletable.org.au/all-things-ethical-eating/what-youcan-do/

79. Agrillo C, Milano S, Roveglia P, Scaffidi C. Slow Food's Contribution to the Debate on the Sustainability of the Food System. Wageningen: European Association of Agricultural Economists; 2015.

80. Oliveira NRF, Jaime PC. O encontro entre o desenvolvimento rural sustentável e a promoção da saúde no Guia Alimentar para a População Brasileira. Saúde Soc 2016; 25:1108-1121.

81. Food and Agriculture Organization of the United Nations (FAO). Plates, pyramids, planet: Developments in national healthy and sustainable dietary guidelines: a state of play assessment. Oxford: The Food Climate Research Network at The University of Oxford; 2016.

82. Lock K, Smith RD, Dangour AD, Keogh-Brown M, Pigatto G, Hawkes C, Fisberg RM, Chalabi Z. Health, agricultural, and economic effects of adoption of healthy diet recommendations. Lancet 2010; 376(9753):1699-1709. 
